\section{Asymmetric dimethylarginine serum levels are associated with early mortality after allogeneic stem cell transplantation}

\author{
Aleksandar Radujkovic, ${ }^{1}$ Hao Dai, ${ }^{2}$ Lambros Kordelas, ${ }^{3}$ Dietrich Beelen, ${ }^{3}$ \\ Sivaramakrishna P. Rachakonda, ${ }^{1,2}$ Carsten Müller-Tidow, ${ }^{1}$ Rajiv Kumar, ${ }^{2}$ \\ Peter Dreger ${ }^{1}$ and Thomas Luft ${ }^{1}$ \\ ${ }^{1}$ Department of Internal Medicine V, University Hospital Heidelberg; ${ }^{2}$ Department of \\ Epidemiology, German Cancer Research Centre (DKFZ), Heidelberg and ${ }^{3}$ Department of \\ Bone Marrow Transplantation, University Hospital Essen, Germany
}

\section{ABSTRACT}

$\tau$ ncreasing evidence suggests that endothelial cell distress is associated with mortality after allogeneic stem cell transplantation and acute graftversus-host disease. Asymmetric dimethylarginine is an endogenous nitric oxide synthase inhibitor that induces endothelial cell dysfunction. We analyzed the impact of pre-transplant serum levels of asymmetric dimethylarginine on outcome after allogeneic stem cell transplantation. Since acute graft-versus-host disease and its treatment are major contributors to posttransplant mortality, the effect of asymmetric dimethylarginine on outcome measures was also assessed after onset of acute graft-versus-host disease. A total of 938 patients allografted at two centers between 2002 and 2013 were included in the retrospective study. In multivariable models, higher pretransplant asymmetric dimethylarginine levels were significantly associated with an increased risk of non-relapse mortality (hazard ratio 1.43 per $1-\log _{2}$ increase, $P=0.005$ ) but not with relapse (hazard ratio 1.21, $P=0.109$ ) within the first year after transplantation. This translated into worse overall survival (hazard ratio $1.45, P<0.0001$ ) and shorter progression-free survival (hazard ratio $1.30, P=0.002$ ) in the first year after transplantation. Higher pre-transplant asymmetric dimethylarginine levels were also associated with shorter overall survival (hazard ratio $1.46, P=0.001$ ) and progressionfree survival (hazard ratio 1.32, $P=0.010$ ) and higher non-relapse mortality (hazard ratio $1.36, P=0.042$ ) within 1 year after the onset of acute graft-versus-host disease. Taken together, our data indicate an association between pre-transplant asymmetric dimethylarginine status and early non-relapse mortality in allografted patients, both overall and after the onset of acute graft-versus-host disease. These findings underline the relevance of endothelial dysfunction for transplant complications.

\section{Introduction}

Non-relapse mortality (NRM) represents a challenge for successful allogeneic stem cell transplantation (SCT). NRM is predominantly driven by graft-versus-host disease (GvHD) as a major complication of allogeneic SCT. There is a body of evidence showing that mortality after allogeneic SCT and after acute GvHD is associated with endothelial cell distress. In previous studies, endothelial markers, such as angiopoietin-2 and nitrates, and, on the genomic level, single nucleotide polymorphisms (SNP) in the recipients' thrombomodulin and CD40 ligand genes, were shown to predict overall mortality and GvHD-related mortality already prior to transplantation..$^{1.5}$

Serum asymmetric dimethylarginine (ADMA) is an endogenous inhibitor of nitric oxide (NO) synthase which competes with arginine and influences the bioavailability of NO., ADMA may cause endothelial dysfunction and, in patients with a compromised vascular system, elevated ADMA levels can predict cardiovascular events and mortality. ${ }^{8-10}$ Recently, two large meta-analyses across different
Ferrata Storti Foundation
Haematologica 2019
Volume 104(4):827-834

\section{Correspondence:}

THOMAS LUFT

thomas.luft@med.uni-heidelberg.de

Received: July 19, 2018.

Accepted: November 23, 2018.

Pre-published: December 4, 2018.

doi:10.3324/haematol.2018.202267

Check the online version for the most updated information on this article, online supplements, and information on authorship \& disclosures: www.haematologica.org/content/104/4/827

(C)2019 Ferrata Storti Foundation

Material published in Haematologica is covered by copyright. All rights are reserved to the Ferrata Storti Foundation. Use of published material is allowed under the following terms and conditions:

https://creativecommons.org/licenses/by-nc/4.0/legalcode. Copies of published material are allowed for personal or internal use. Sharing published material for non-commercial purposes is subject to the following conditions:

https://creativecommons.org/licenses/by-nc/4.0/legalcode, sect. 3. Reproducing and sharing published material for com mercial purposes is not allowed without permission in writing from the publisher. 
patient and general populations demonstrated that circulating $\mathrm{ADMA}$ is also an independent risk factor for allcause mortality. ${ }^{11,12}$ In view of these findings, the present retrospective study investigated the impact of pre-transplant serum ADMA levels on major outcome measures in the setting of allogeneic SCT.

\section{Methods}

\section{Patients}

Adult patients (age $\geq 18$ years) who were allografted between 2002 and 2013 at two centers (Heidelberg, n=518, and Essen, $\mathrm{n}=420$ ) and who had serum samples available for ADMA measurement (collected directly before the start of conditioning chemotherapy prior to allogeneic SCT) were included in this study. Written informed consent to sample and data collection according to the Declaration of Helsinki was obtained from all patients, and the local ethics committees approved the study.

\section{Assessment of serum levels of asymmetric dimethyl- arginine and endothelium-related serum factors}

Serum levels of ADMA and endothelium-related serum factors were assessed by enzyme-linked immunosorbent assay retrospectively in the last serum sample taken before the start of the conditioning treatment.

\section{Statistical analysis}

Overall survival (OS), progression-free survival (PFS), time to relapse, and NRM (death in the absence of prior relapse) were calculated from the date of allogeneic SCT to the appropriate endpoint. PFS was defined as the time from transplantation to relapse of the underlying disease or death. Since acute GvHD and its treatment are major contributors to post-transplant mortality, OS, PFS and NRM were also assessed after acute GvHD (i.e. from the date of onset of acute GvHD). NRM and recurrence of the underlying malignancy were considered as competing events. For the incidence of acute GvHD, the competing events were relapse and death in remission without acute GvHD. The Kaplan-Meier method was used to estimate distributions of survival times. Follow-up times were calculated by the reverse Kaplan-Meier estimate. ${ }^{13}$ Cumulative incidence functions were implemented to account for the competing risks,

Due to the relatively wide range of supposedly normal ADMA serum levels, pre-transplant ADMA was analyzed as a continuous variable. Since ADMA serum levels did not follow a normal distribution and the data were $\log _{2}$-transformed, the prognostic effect of pre-transplant ADMA on OS, PFS, relapse, and NRM as a continuous variable was evaluated using Cox regression models. Cox proportional hazards regression modeling was used for OS and PFS (overall and after the onset of acute GvHD). Relapse and NRM (overall and after the onset of acute GvHD) were analyzed by cause-specific Cox models.

To illustrate the continuous effect of pre-transplant ADMA on the different endpoints, patients were grouped according to ADMA quartiles, and the observation period was restricted to the first year after transplantation or acute GvHD.

To assess the dependency of endothelium-related serum markers [free interleukin (IL)-18, sCD141, and nitrates] on ADMA levels, the Jonckheere trend test was applied comparing serum marker data in four increasing intervals of pre-transplant ADMA levels determined by the quartiles of the ADMA distribution. The Jonckheere trend test is a rank-based nonparametric test which is used to test for an ordered difference in medians. ${ }^{14}$

All statistical tests were two-sided. Hazard ratios (HR) were estimated with $95 \%$ confidence intervals (95\% CI). $P$ values $<0.05$ were considered statistically significant.

Details regarding prophylaxis, diagnosis, and treatment of $\mathrm{GvHD}$, the assessment of serum levels of ADMA and endothelium-related serum factors, SNP analyses in the inducible nitric oxide synthase gene (INOS) and additional statistical methods are described in the Online Supplement.

\section{Results}

\section{Patients', disease and treatment characteristics and} graft-versus-host disease incidence

A total of 938 patients met the eligibility criteria for this study. The patients', disease and transplant characteristics are given in Table 1. A total of 493 patients died after allogeneic SCT, of whom 309 died within the first year after transplantation. The cumulative incidences of relapse and NRM at 1 year after transplantation were $23.0 \%$ (95\% CI: $20.3 \%-25.7 \%$ ) and $18.2 \%$ (95\% CI: $15.8 \%-20.7 \%$ ), respectively. The cumulative incidences of acute GvHD

Table 1. Patients', disease and transplant characteristics.

\begin{tabular}{|c|c|}
\hline Parameter & $\mathrm{N}=938$ \\
\hline Age [in years] at allogeneic SCT, median, (IQR) & $54(46-62)$ \\
\hline $\begin{array}{l}\text { Recipient sex, n (\%) } \\
\text { Female } \\
\text { Male }\end{array}$ & $\begin{array}{l}424(45) \\
514(55)\end{array}$ \\
\hline $\begin{array}{l}\text { Disease, n (\%) } \\
\text { Myeloid }^{\mathrm{a}} \\
\text { Lymphoid }^{\mathrm{b}}\end{array}$ & $\begin{array}{l}665(71) \\
273(29)\end{array}$ \\
\hline $\begin{array}{l}\text { Disease stage at allogeneic } \mathrm{SCT}^{\mathrm{c}} \text {, n (\%) } \\
\text { Early } \\
\text { Late/intermediate }\end{array}$ & $\begin{array}{l}306(33) \\
632(67)\end{array}$ \\
\hline $\begin{array}{l}\text { Conditioning }^{\mathrm{d}} \text { regimen, } \mathrm{n}(\%) \\
\text { Reduced intensity } \\
\text { Myeloablative }\end{array}$ & $\begin{array}{l}804(86) \\
134(14)\end{array}$ \\
\hline $\begin{array}{l}\text { Stem cell source, } \mathrm{n}(\%) \\
\text { Peripheral blood } \\
\text { Bone marrow }\end{array}$ & $\begin{array}{c}861(92) \\
77(8)\end{array}$ \\
\hline $\begin{array}{l}\text { Donor type, n (\%) } \\
\text { Unrelated } \\
\text { Related }\end{array}$ & $\begin{array}{l}700(75) \\
238(25)\end{array}$ \\
\hline $\begin{array}{l}\text { Donor-recipient HLA-matching, n (\%) } \\
\text { Mismatched }\end{array}$ & $242(26)$ \\
\hline
\end{tabular}

$\begin{array}{ll}\text { Matched } & 696(74)\end{array}$

Antithymocyte globulin treatment, n (\%)

No 225 (24)

Yes

$\begin{array}{ll}\text { Donor sex, } \mathrm{n}(\%) & 311(33) \\ \text { Female } & 627(67) \\ \text { Male } & \end{array}$

Pre-transplant ADMA,$\mu \mathrm{M}$, median (IQR) $\quad 0.73(0.59-0.97)$

Pre-transplant nitrate levels, $\mu \mathrm{M}$, median (IQR) 22.4 (15.0-35.7)

Pre-transplant free IL-18, pg/mL, median (IQR) 442 (316-683)

Pre-transplant soluble CD141 , pg/mL, median (IQR) 4179 (3386-5376)

ADMA: asymmetric dimethylarginine; SCT: stem cell transplantation; HLA: human leukocyte antigen; IL-18: interleukin 18; IQR, interquartile range; ${ }^{M}$ Meloid: acute myeloid leukemia, myelodysplastic and myeloproliferative syndromes. ${ }^{\circ} L y m p h o i d$ acute lymphoblastic leukemia, chronic lymphocytic leukemia, lymphoma and multi-

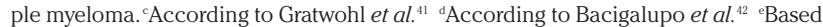

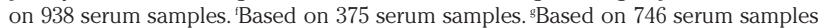
hBased on 776 serum samples. 
(any grade) and grade 3-4 acute GvHD on day +100 after transplantation were $54.0 \%$ (95\% CI: 50.8\%-57.3\%) and $13.7 \%$ (95\% CI: $11.1 \%-16.3 \%)$, respectively. The estimated median follow-up time was 64.9 months (95\% CI: 62.0-67.9; range, 0.0-167.7).

\section{Pre-transplant serum asymmetric dimethylarginine levels and their impact on outcome measures after allogeneic stem cell transplantation}

Across all 938 patients, the median pre-transplant ADMA level was $0.73 \mu \mathrm{M}$ [interquartile range (IOR) 0.590.97]. Initial analyses indicated that the proportional hazards assumption was violated for pre-transplant ADMA, i.e., the effect of pre-transplant ADMA on mortality risks was not constant over time and was limited to the first year after transplantation or after acute GvHD (additional statistical methods are given in the Online Supplement).

In multivariable analyses adjusted for known confounders, increasing pre-transplant ADMA levels, as a continuous variable, were significantly associated with worse OS (HR 1.45 per $1-\log _{2}$ increase, $95 \%$ CI: 1.21-1.74, $P<0.0001$ ) and shorter PFS (HR 1.30, 95\% CI: 1.10-1.54, $P=0.002$ ) within the first year after allogeneic SCT, but not thereafter. This was based on an increased risk of NRM (HR 1.43, 95\% CI: 1.12-1.83, $P=0.005$ ) rather than relapse (HR 1.21, 95\% CI: $0.96-1.52, P=0.109$ ) in the year following transplantation. The results of the multivariable analyses with the endpoints OS, PFS, NRM and post-transplant relapse are summarized in Table 2.

Increasing pre-transplant $\mathrm{ADMA}$ levels were not signif- icantly associated with the incidence of acute GvHD (univariable: any grade, HR 1.04, 95\% CI: 0.90-1.20, $P=0.583$; univariable: grade $3-4, \mathrm{HR} 1.27,95 \%$ CI: $0.93-1.73$, $P=0.129)$. However, we observed an association with higher risk of NRM after GvHD onset (HR 1.36, 95\% CI: 1.01-1.83, $P=0.042$ ). This translated into worse OS (HR 1.46, 95\% CI: 1.17-1.83, $P=0.001)$ and shorter PFS (HR $1.32,95 \%$ CI: $1.07-1.63, P=0.010)$ within the first year after the onset of acute GvHD. The results of the multivariable analyses with the endpoints OS, PFS, and NRM after acute GvHD are given in Table 3.

The association of increasing pre-transplant ADMA levels with shorter OS and PFS and higher risk of NRM in the multivariable models, both within the first year aftertransplantation and within the first year after the development of acute GvHD, could also be observed when patients were stratified according to the transplant center applying a meta-analytic approach (Figure 1).

In order to illustrate the continuous effect of pre-transplant ADMA levels on all aforementioned endpoints, patients were grouped according to four increasing ranges of pre-transplant ADMA levels determined by the quartiles of the ADMA distribution. The corresponding plots showing OS, PFS, NRM and relapse in the first year after transplantation, and OS, PFS, and NRM in the first year after acute GvHD are given in Figure 2.

In addition, the prediction performance of the univariable ADMA models was assessed with regards to OS and PFS (overall and after acute GvHD). As compared to the respective Kaplan-Meier survival estimates, the ADMA

Table 2. Multivariable analysis of predictors of overall survival, progression-free survival, non-relapse mortality and relapse ( $\mathrm{n}=938$, complete case analysis).

\begin{tabular}{|c|c|c|c|c|c|c|c|c|}
\hline \multirow[b]{2}{*}{ Covariate } & \multicolumn{2}{|c|}{ OS } & \multicolumn{2}{|c|}{ PFS } & \multicolumn{2}{|c|}{ NRM* } & \multicolumn{2}{|c|}{ Relapse* } \\
\hline & HR (95\% Cl) & $P$ & HR (95\% Cl) & $P$ & HR (95\% Cl) & $P$ & HR (95\% CI) & $P$ \\
\hline $\begin{array}{l}\text { ADMA (per 1- } \log _{2} \text { increase): } \\
1^{\text {st year }}\end{array}$ & $1.45(1.21-1.74)$ & $<0.0001$ & $1.30(1.10-1.54)$ & 0.002 & $1.43(1.12-1.83)$ & 0.005 & $1.21(0.96-1.52)$ & 0.109 \\
\hline $\begin{array}{l}\text { ADMA (per 1- } \log _{2} \text { increase): } \\
2-3 \text { years }\end{array}$ & $1.02(0.74-1.41)$ & 0.888 & $1.00(0.71-1.42)$ & 0.980 & $1.18(0.69-2.04)$ & 0.543 & $0.92(0.59-1.42)$ & 0.694 \\
\hline $\begin{array}{l}\text { ADMA (per 1- } \log _{2} \text { increase): } \\
\text { after } 3 \text { years }\end{array}$ & $1.03(0.69-1.55)$ & 0.878 & $0.93(0.58-1.52)$ & 0.781 & $0.87(0.48-1.58)$ & 0.645 & $1.07(0.47-2.44)$ & 0.877 \\
\hline Age at transplantation (per year) & $1.01(1.00-1.02)$ & 0.008 & $1.01(1.00-1.02)$ & 0.034 & $1.03(1.01-1.04)$ & $<0.0001$ & $1.00(0.96-1.01)$ & 0.395 \\
\hline $\begin{array}{l}\text { Donor sex } \\
\text { Female } \\
\text { Male }\end{array}$ & $\begin{array}{c}\text { Ref } \\
0.82(0.68-1.00)\end{array}$ & 0.046 & $\begin{array}{c}\text { Ref } \\
0.83(0.72-1.04)\end{array}$ & 0.113 & $\begin{array}{c}\text { Ref } \\
0.76(0.58-0.99)\end{array}$ & 0.043 & $\begin{array}{c}\text { Ref } \\
0.96(0.75-1.23)\end{array}$ & 0.737 \\
\hline $\begin{array}{l}\text { Recipient sex } \\
\text { Female } \\
\text { Male }\end{array}$ & $\begin{array}{c}\text { Ref } \\
1.10(0.92-1.32)\end{array}$ & 0.298 & $\begin{array}{c}\text { Ref } \\
1.06(0.89-1.26)\end{array}$ & 0.507 & $\begin{array}{c}\text { Ref } \\
1.14(0.87-1.48)\end{array}$ & 0.346 & $\begin{array}{c}\text { Ref } \\
0.99(0.78-1.25)\end{array}$ & 0.937 \\
\hline $\begin{array}{l}\text { Donor-recipient HLA matching } \\
\text { Mismatched } \\
\text { Matched }\end{array}$ & $\begin{array}{c}\text { Ref } \\
0.67(0.55-0.81)\end{array}$ & $<0.0001$ & $\begin{array}{c}\text { Ref } \\
0.74(0.62-0.90)\end{array}$ & 0.002 & $\begin{array}{c}\text { Ref } \\
0.55(0.42-0.72)\end{array}$ & $<0.0001$ & $\begin{array}{c}\text { Ref } \\
0.98(0.75-1.28)\end{array}$ & 0.895 \\
\hline $\begin{array}{l}\text { Disease stage }^{\dagger} \\
\text { Early } \\
\text { Late/intermediate }\end{array}$ & $\begin{array}{c}\text { Ref } \\
1.18(0.97-1.43)\end{array}$ & 0.090 & $\begin{array}{c}\text { Ref } \\
1.19(0.99-1.44)\end{array}$ & 0.060 & $\begin{array}{c}\text { Ref } \\
0.99(0.75-1.29)\end{array}$ & 0.912 & $\begin{array}{c}\text { Ref } \\
1.43(1.11-1.86)\end{array}$ & 0.006 \\
\hline $\begin{array}{l}\text { Conditioning } \\
\text { Myeloablative } \\
\text { Reduced intensity } \\
\end{array}$ & $\begin{array}{c}\text { Ref } \\
0.96(0.72-1.28) \\
\end{array}$ & 0.785 & $\begin{array}{c}\text { Ref } \\
1.00(0.76-1.31) \\
\end{array}$ & 0.972 & $\begin{array}{c}\text { Ref } \\
0.87(0.57-1.36)\end{array}$ & 0.559 & $\begin{array}{c}\text { Ref } \\
1.06(0.74-1.52) \\
\end{array}$ & 0.749 \\
\hline
\end{tabular}

Number of events: overall survival (OS), $\mathrm{n}=493$; progression-free survival (PFS), $\mathrm{n}=540$; non-relapse mortality (NRM), $\mathrm{n}=243$; relapse, $\mathrm{n}=297 .{ }^{*}$ Cause-specific hazards from a competing risks analysis for relapse and NRM. ${ }^{\dagger}$ According to Gratwohl et al. ${ }^{41}{ }^{\ddagger}$ According to Bacigalupo et al. ${ }^{42}$ ADMA: asymmetric dimethylarginine; CI: confidence interval; HLA: human leukocyte antigen; HR: hazard ratio. 
models showed a slightly improved prediction performance. The corresponding prediction error curves representing the Brier score over time are depicted in Online Supplementary Figure S1.

Association of asymmetric dimethylarginine levels with endothelium-related serum factors and high pre-transplant levels in the context of INOS polymorphisms

Samples for measurement of serum concentrations of free IL-18, soluble thrombomodulin (sCD141), and nitrates were available for 746, 776 and 375 patients, respectively. The corresponding median pre-transplant serum levels are given in Table 1. Pre-transplant ADMA levels correlated with serum levels of nitrates, free IL-18, and sCD141 (Figure 3A-C).

The concept of endothelial vulnerability involves a proinflammatory environment and ADMA is able to modify $\mathrm{NO}$ synthase and, in particular, inducible NO synthase (iNOS) activity. ${ }^{7,15}$ We analyzed whether polymorphisms in INOS modulated the observed effects of ADMA. Genotype data on INOS SNP were available for a total of 386 patients. In order to evaluate the effect of ADMA in the context of INOS polymorphisms, an optimal cut-off with regard to $O S$ in the first year after transplantation was determined, yielding multiple cut-points (maxima) (Online Supplementary Figure S2). The maximum at 0.97 $\mu \mathrm{M}$, which also represents the upper quartile of the ADMA distribution, was chosen to stratify patients into groups with high $(\geq 0.97 \mu \mathrm{M})$ or low $(<0.97 \mu \mathrm{M})$ ADMA levels in the context of INOS polymorphisms. There was no substantial influence of INOS SNP on the effect of ADMA on OS or NRM within the first year after allogeneic SCT (Online Supplementary Figures S3-5).

\section{Discussion}

To the best of our knowledge, this is the first report of an association between ADMA levels measured prior to transplant and subsequent risk of mortality in allografted patients. ADMA is a well-characterized cardiovascular risk factor, and dysfunction of the endothelium appears to be a common finding in studies investigating the role of $\mathrm{ADMA}$ in (cardio-)vascular disease. ${ }^{10}$ Elevated ADMA levels have also been reported in autoimmune diseases, linking inflammation with autoimmunity-related vascular complications. ${ }^{16}$ In renal transplant recipients, associations of increased ADMA levels with acute rejection and mortality have been observed. ${ }^{17,18}$ Furthermore, high ADMA levels were associated with pro-inflammatory conditions and were shown to predict mortality risk in critically ill and septic patients. ${ }^{19,20}$ Finally, ADMA was strongly associated with all-cause mortality across different patient and general populations in recent meta-analyses. ${ }^{11,12}$

Previous studies by us and others ${ }^{2-5,21-23}$ led us to propose the hypothesis of "endothelial vulnerability" as an important contributor to the main complications of allogeneic SCT. This concept may explain why a proportion of patients with acute GvHD fail to respond to escalating immunosuppressive therapy and ultimately succumb to acute GvHD, its treatment and/or related complications. ${ }^{21}$ It denotes a risk that, in the setting of allogeneic SCT, is substantiated particularly in the presence of additional challenges, i.e., conditioning treatment and/or allogeneic T-cell attacks in a pro-inflammatory environment, and may result in endothelial damage that leads to perpetuating inflammatory end-organ destruction despite (initially) effective control of T-cell activity. Since this "vulnerability" is, at least in

Table 3. Multivariable analysis of predictors of overall survival, progression-free survival and non-relapse mortality after onset of acute graft-versus-host disease (complete case analysis).

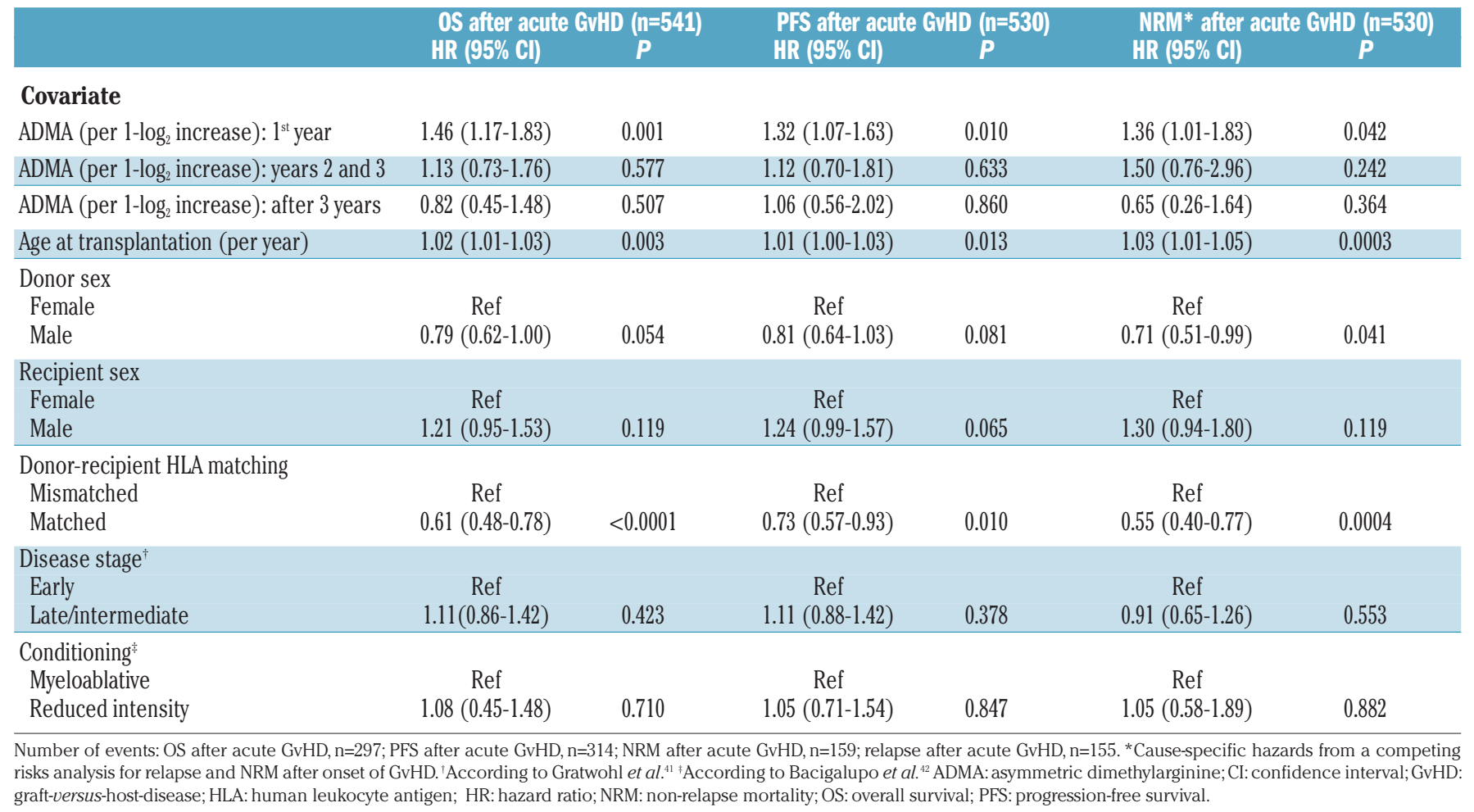


part, a characteristic of the recipient's endothelial cell system, increased risk of mortality, both overall and after the onset of acute GvHD, may be determined (and predicted) already prior to transplantation. However, it should be noted, that, although most markers of "endothelial vulnerability" are likely to predict outcome after a severe challenge, particularly after acute $\mathrm{GvHD}$, they do not seem to be associated with a higher incidence of acute GvHD. In line with this, in the present study, pre-transplant ADMA was not a biomarker of acute GvHD risk but was associated with early NRM, both overall and following acute GvHD, which translated into shorter survival outcomes.

With regards to the endothelial system, higher ADMA levels correlated positively with endothelium-related serum factors, such as sCD141, free IL-18, and nitrates. Thrombomodulin is a surface receptor protein released/shed from the endothelium in an extracellular soluble form (sCD141) during cell distress and elevated serum levels are frequently indicative of inflammatory cell damage. $^{24}$ IL-18 is a pleiotropic, pro-inflammatory cytokine associated with coronary heart disease $e^{25}$ and de-regulation of the IL-18/IL-18BP pathway ${ }^{26}$ was shown to cause endothelial cell dysfunction. Interestingly, pre-transplant nitrate levels, which are markers of in vivo NO production, also correlated positively with ADMA, which is a NO synthase inhibitor. This seemingly contradictory finding is in line with some but not all observations made in other clinical settings. ${ }^{27,28}$

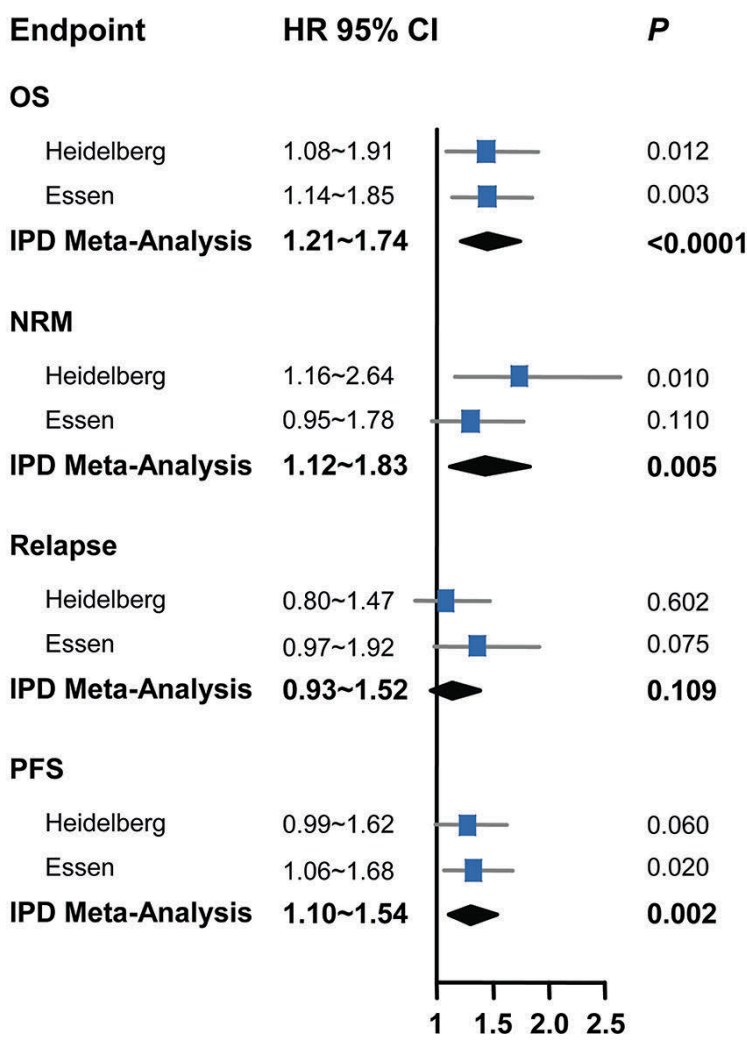

In this context, it should be noted, that higher pre-transplant nitrates levels were also associated with a higher incidence of NRM in a previous study. ${ }^{4}$ In pro-inflammatory conditions, large amounts of $\mathrm{NO}$ are produced by iNOS in numerous cell types. ${ }^{29}$ In the cardiovascular system, upregulation of iNOS may contribute to endothelial dysfunction. ${ }^{30}$

In the present study, however, INOS SNP did not influence the effect of ADMA on mortality. Certainly, interactions of $\mathrm{ADMA}$ with other $\mathrm{NO}$ synthase family members cannot be excluded, and there is also evidence that NO synthase-independent mechanisms may contribute to the detrimental biological effects associated with increases in ADMA. ${ }^{31,32}$ Consequently, in the setting of allogeneic SCT, elevated levels of both ADMA and nitrate prior to conditioning and immunosuppressive therapy are likely to reflect compromised endothelial homeostasis in the recipient which, particularly in the context of a severe challenge, such as acute GvHD, may result in an impaired outcome. However, since GvHD is an immune-triggered process, an influence of the donor's ADMA or nitrate levels on the intensity of the subsequent allogeneic immune response in the recipient cannot definitely be ruled out.

The median pre-transplant ADMA level in our series was $0.73 \mu \mathrm{M}$ which is in accordance with literature data that suggest normal serum ADMA levels are in the range of 0.25-0.92 $\mu \mathrm{M}$ when measured by an enzyme-linked immunosorbent assay. ${ }^{33}$ Importantly, since ADMA is direct-
Endpoint $\quad$ HR $95 \% \mathrm{Cl}$

$\boldsymbol{P}$

OS after acute GVHD

\begin{tabular}{|c|c|c|c|}
\hline Heidelberg & $1.09 \sim 2.39$ & -1 & 0.012 \\
\hline Essen & $1.03 \sim 1.81$ & - & 0.032 \\
\hline IPD Meta-Analysis & $1.17 \sim 1.83$ & 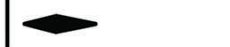 & 0.001 \\
\hline \multicolumn{4}{|c|}{ NRM after acute GVHD } \\
\hline Heidelberg & $1.00 \sim 2.86$ & $H_{-1}$ & 0.051 \\
\hline Essen & $0.84 \sim 1.78$ & 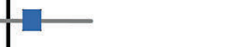 & 0.287 \\
\hline IPD Meta-Analysis & $1.01 \sim 1.83$ & $K$ & 0.042 \\
\hline \multicolumn{4}{|c|}{ PFS after acute GVHD } \\
\hline Heidelberg & $0.89 \sim 1.85$ & & 0.180 \\
\hline Essen & $0.99 \sim 1.69$ & -1 & 0.055 \\
\hline IPD Meta-Analysis & $1.07 \sim 1.63$ & & 0.010 \\
\hline
\end{tabular}

Figure 1. Higher pre-transplant asymmetric dimethylarginine levels are associated with non-relapse mortality and worse overall and progression-free survival in the first year after transplantation and after acute graft-versus-host disease. Meta-analyses of the multivariable effect of pre-transplant asymmetric dimethylarginine (ADMA) levels as a continuous variable (per 1- $\log _{2}$ increase) for overall survival (OS), progression-free survival (PFS), and non-relapse mortality (NRM) in the first year after transplantation and after acute graft-versus-host disease (GvHD), and relapse in the first year after allografting. CI: confidence interval; HR: hazard ratio; IPD: individual patient data. 
A

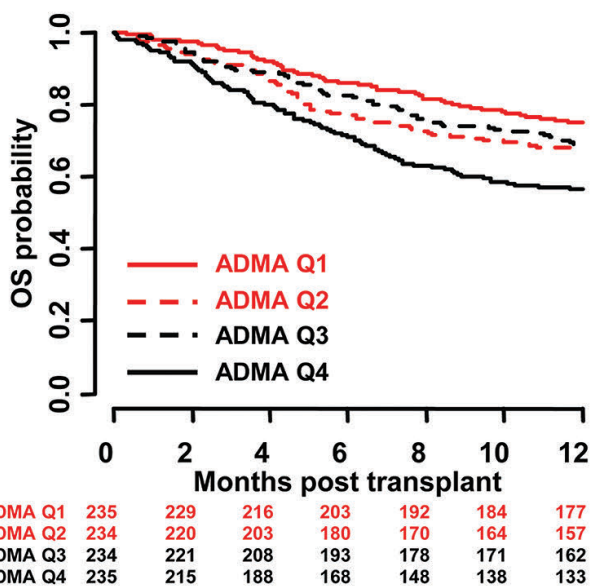

C

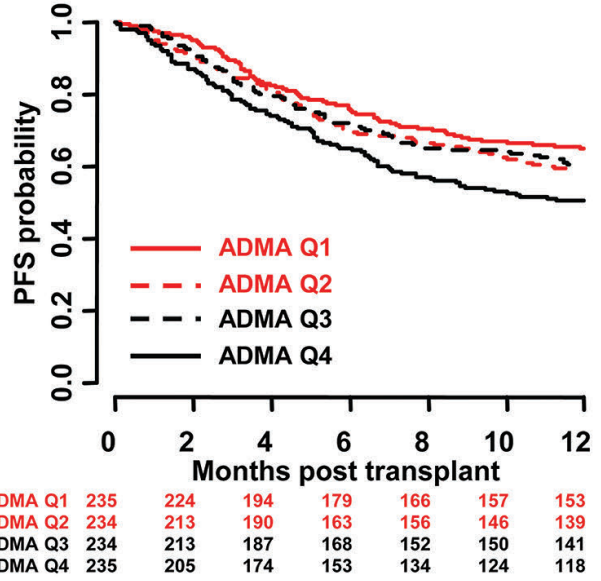

$\mathrm{E}$

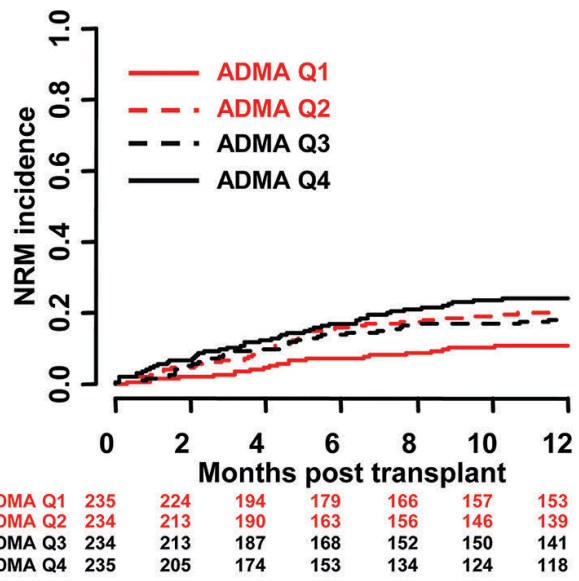

G

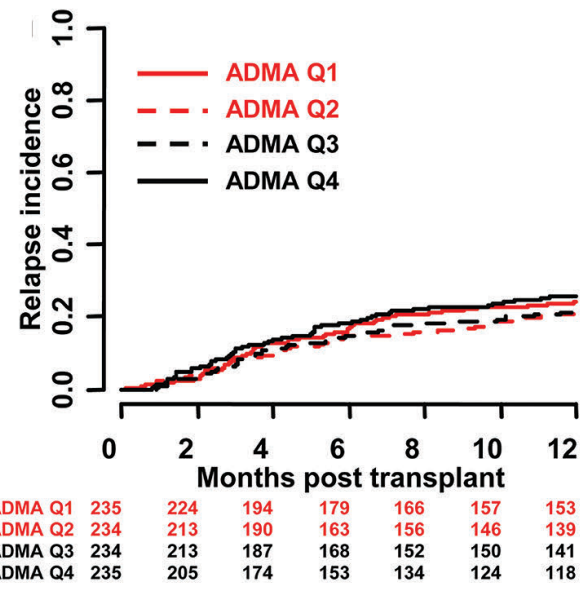

B

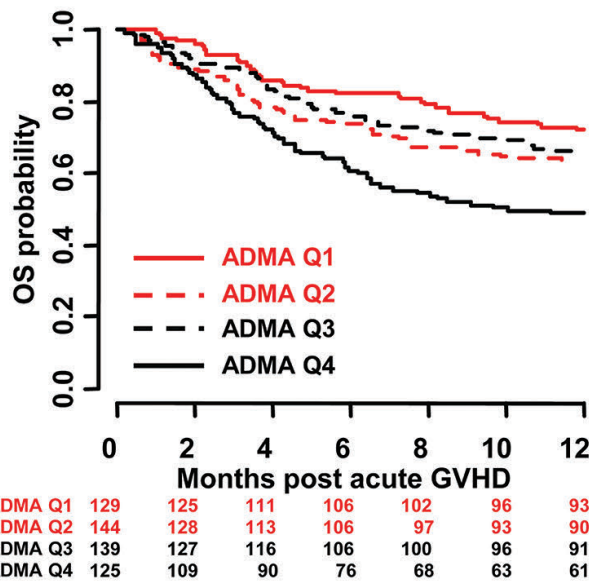

D

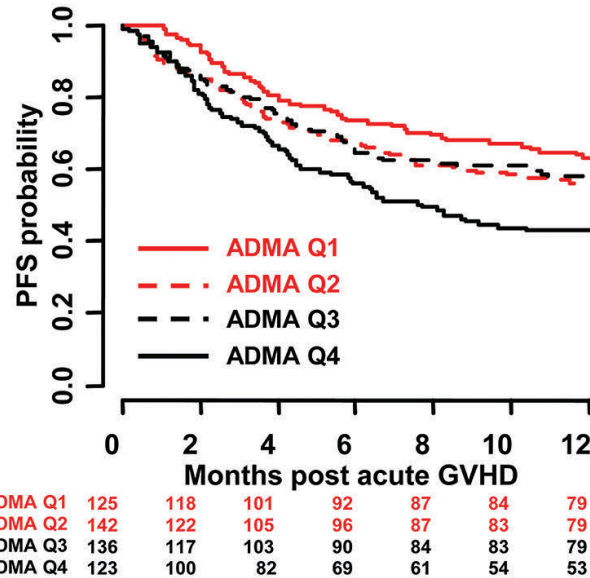

$\mathrm{F}$

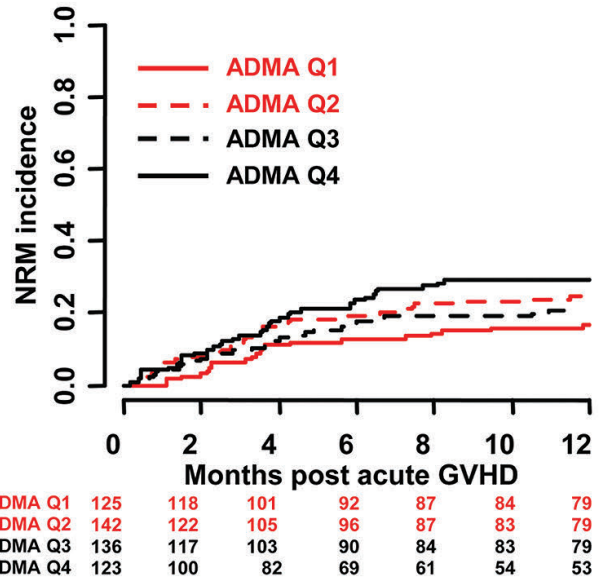

Figure 2. Outcome of patients in the first year after allogeneic stem cell transplantation and after onset of acute graft-versus-host disease when stratified according to pre-transplant asymmetric dimethylarginine quartiles. (A, B) Higher pre-transplant asymmetric dimethylarginine (ADMA) serum levels were associated with lower probabilities of overall survival (OS) in (A) the first year after allogeneic stem cell transplantation and (B) after the onset of acute graft-versus-host disease (GvHD). (C, D) The probabilities of progression-free survival (PFS) in (C) the first year after transplantation and (D) after the onset of acute GvHD were substantially lower in patients with higher pre-transplant ADMA levels. (E, F) Higher pre-transplant ADMA serum levels were associated with higher incidences of non-relapse mortality (NRM) in (E) the first year after allografting and (F) after the onset of acute GvHD. (G) In contrast, the ncidence of relapse during the first post-transplant year was not affected by pre-transplant ADMA levels. Note: Quartiles were chosen for reasons of visualization of the continuous ADMA effect which was observed for every 2-fold change in multivariable analyses (Tables 2 and 3). For this reason, and since pre-transplant ADMA values were not normally distributed, there is an overlap of the second and third quartile. Q1-4, quartiles of the ADMA distribution. 
A

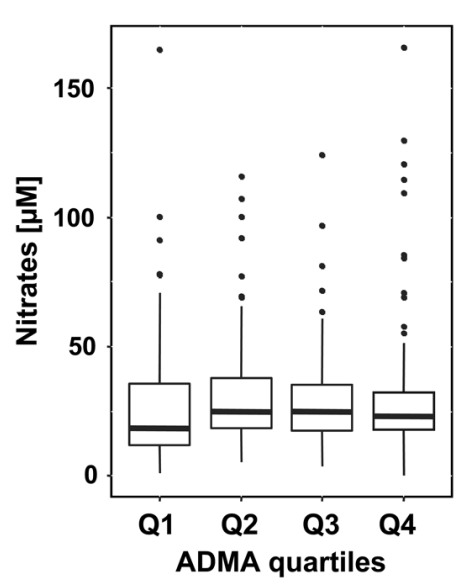

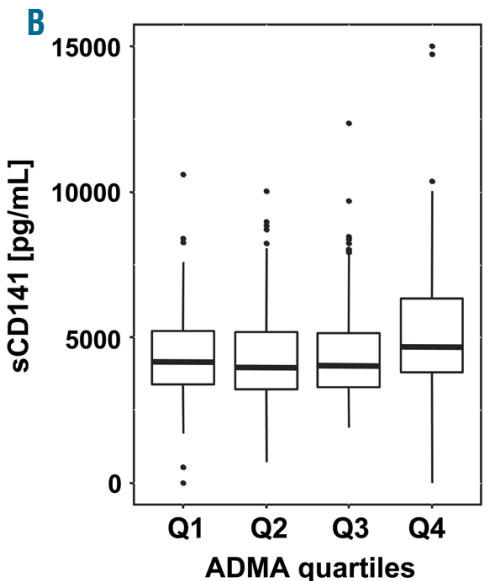

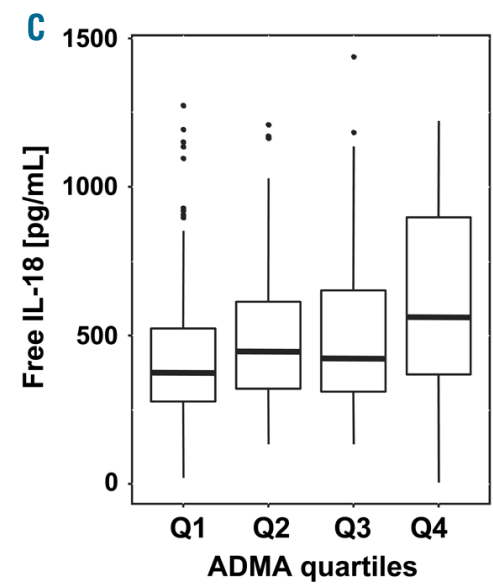

Figure 3. Relationship between quartiles of pre-transplant asymmetric dimethylarginine serum concentration and serum concentration of nitrates, free interleukin-18, and soluble thrombomodulin (sCD141). Trend analysis showed that pre-transplant concentrations of $(A)$ nitrates $(n=375$, (B) soluble thrombomodulin $(n=776)$ and $(C)$ free interleukin-18 $(n=746)$ were positively correlated with quartiles of pre-transplant ADMA concentration $(J o n c k h e e r e$ trend test, $P=0.010$, $P<0.0001$ and $P<0.0001$, respectively). ADMA, asymmetric dimethylarginine; IL-18: free interleukin-18; 01-4, quartiles 1-4 of the ADMA distribution; sCD141: soluble thrombomodulin.

ly involved in endothelial dysfunction, it may be a therapeutic target. However, a specific ADMA-lowering agent is not yet available. ${ }^{34}$ Supplementation of L-citrulline may be employed to boost intracellular arginine levels and to reduce ADMA production. In a few clinical studies, supplemental L-citrulline in multi-gram doses was well tolerated and was shown to exert various effects suggestive of cardiovascular protection; it could, therefore, be studied in high-risk patients with elevated ADMA. ${ }^{35,36}$ Given the continuous relation between ADMA serum levels and survival outcomes in our study, treatment of patients irrespective of the individual's pre-transplant ADMA status may be favored and such a clinical study should ideally be placebocontrolled. However, given the necessity of oral L-citrulline ingestion in multi-gram doses (3-10 g/day) and possible drug interactions ${ }^{36}$ the first interventional study in the setting of allogeneic SCT setting may rather enroll patients with markedly elevated pre-transplant ADMA serum concentrations, who are at high risk of early mortality according to our retrospective analysis. Based on literature data and our results, in a possible clinical trial setting, ADMA levels $\geq 1 \mu \mathrm{M}$ could be used to define such a high-risk population of patients.

Besides its retrospective nature, some limitations of our study need to be addressed. ADMA was measured at a single time point and thus no information on the serum kinetics of ADMA levels in the post-transplant period can be provided. Furthermore, with regard to the known association of ADMA with cardiovascular pathologies, one could ask whether the NRM events in our study were related to cardiovascular disease. However, it should be noted that in the absence of disease recurrence, the exact cause of death in allografted patients, particularly in the early post-transplant period, is often difficult to determine and for the purposes of categorization, infection, GvHD and (general) organ failure are generally used. In a recently published large registry study, ${ }^{37}$ the main causes of NRM were (in descending order): infection, GvHD and respiratory disease, all of which may be linked to endothelial distress. The fre- quency of (isolated) cardiovascular events after transplantation is rather low with a cumulative incidence of $6 \%$ after 15 years. ${ }^{38}$ However, it should be taken into account that endothelial factors play a crucial role in many important complications of allogeneic SCT, such as transplant-associated microangiopathy, veno-occlusive disease, and refractory GvHD. ${ }^{1,39,40}$ Finally, like most clinical research on ADMA, our data are observational, describe a relationship and do not allow for interpretation of causality. Our results need to be validated, ideally in a prospective interventional study. As already pointed out above, investigating the effects of $\mathrm{L}$ citrulline treatment in high-risk patients undergoing allogeneic SCT may be a feasible approach.

In summary, this retrospective study shows an association between ADMA levels and outcome after allogeneic SCT. Higher ADMA levels were a risk factor for early mortality in allografted patients, both overall and after the onset of acute $\mathrm{GvHD}$, and were correlated with other biomarkers of endothelial vulnerability. Further studies on the role of the endothelium and markers associated with endothelial distress in the setting of allogeneic SCT are warranted.

\section{Acknowledgments}

The authors would like to thank Michael Hess and Alexandra Hof for their excellent technical assistance and construction of the tissue bank, Maria Gawlik for assistance in the collection of clinical data, and Thorsten Brenner and Stefan Hofer (Department of Anesthesiology, University of Heidelberg) for helpful discussions. We also wish to acknowledge the excellent help of Axel Benner (Department of Biostatistics, German Cancer Research Center) regarding the statistical analyses.

\section{Funding}

This work was supported by grants from the HelmholtzAlliance on Immunotherapy of Cancer, B.L.U.T. e.V. (Weingarten, Germany) and the Wilhelm-Sander-Stiftung (grant $n$. 2008.068.1). HD was sponsored by the China Scholarship Council. 


\section{References}

1. Carreras E, Diaz-Ricart M. The role of the endothelium in the short-term complications of hematopoietic SCT. Bone Marrow Transplant. 2011:46(12):1495-1502.

2. Dietrich S, Falk CS, Benner A, et al. Endothelial vulnerability and endothelial damage are associated with risk of graft-versus-host disease and response to steroid treatment. Biol Blood Marrow Transplant. 2013;19(1):22-27.

3. Tatekawa S, Kohno A, Ozeki K, et al. A novel diagnostic and prognostic biomarker panel for endothelial cell damage-related complications in allogeneic transplantation. Biol Blood Marrow Transplant. 2016;22(9): 1573-1581.

4. Dietrich S, Okun JG, Schmidt K, et al. High pre-transplant serum nitrate levels predict risk of acute steroid-refractory graft-versushost disease in the absence of statin therapy. Haematologica. 2014;99(3):541-547.

5. Rachakonda SP, Penack O, Dietrich S, et al. Single-nucleotide polymorphisms within the thrombomodulin gene (THBD) predict mortality in patients with graft-versus-host disease. J Clin Oncol. 2014;32(30):3421-3427.

6. Vallance P, Leone A, Calver A, Collier J, Moncada S. Endogenous dimethylarginine as an inhibitor of nitric oxide synthesis. J Cardiovasc Pharmacol. 1992;20 (Suppl 12):S60-62

7. Vallance P, Leone A, Calver A, Collier J, Moncada S. Accumulation of an endogenous inhibitor of nitric oxide synthesis in chronic renal failure. Lancet. 1992;339 (8793):572-575.

8. Kielstein IT, Impraim B, Simmel S, et al. Cardiovascular effects of systemic nitric oxide synthase inhibition with asymmetrical dimethylarginine in humans. Circulation. 2004;109(2):172-177.

9. Böger RH. Asymmetric dimethylarginine, an endogenous inhibitor of nitric oxide synthase, explains the "L-arginine paradox" and acts as a novel cardiovascular risk factor. J Nutr. 2004:134:(10 Suppl):2842S-2847S

10. Bouras G, Deftereos S, Tousoulis D, et al. Asymmetric dimethylarginine (ADMA): a promising biomarker for cardiovascular disease? Curr Top Med Chem. 2013;13(2):180200.

11. Schlesinger S, Sonntag SR, Lieb W, Maas R. Asymmetric and symmetric dimethylarginine as risk markers for total mortality and cardiovascular outcomes: a systematic review and meta-analysis of prospective studies. PLoS One. 2016;11(11): e0165811.

12. Zhou S, Zhu O, Li X, et al. Asymmetric dimethylarginine and all-cause mortality: a systematic review and meta-analysis. Sci Rep. 2017;7:44692.

13. Schemper M, Smith TL. A note on quantifying follow-up in studies of failure time. Control Clin Trials. 1996;17(4):343-346.

14. Jonckheere AR. A distribution free k-sample test against ordered alternatives. Biometrika. 1954(1-2):133-145
15. Tsikas D, Bollenbach A, Hanff E, Kayacelebi AA. Asymmetric dimethylarginine (ADMA), symmetric dimethylarginine (SDMA) and homoarginine (hArg): the ADMA, SDMA and hArg paradoxes. Cardiovasc Diabetol. 2018;17(1):1.

16. Chen XM, Hu CP, Li YJ, Jiang JL. Cardiovascular risk in autoimmune disorders: role of asymmetric dimethylarginine. Eur J Pharmacol. 2012;696(1-3):5-11.

17. Esposito C, Grosjean F, Torreggiani M, et al. Increased asymmetric dimethylarginine serum levels are associated with acute rejection in kidney transplant recipients. Transplant Proc. 2009;41(5):1570-1573.

18. Frenay AR, van den Berg E, de Borst MH, et al. Plasma ADMA associates with all-cause mortality in renal transplant recipients. Amino Acids. 2015:47(9):1941-1949.

19. Koch A, Weiskirchen R, Kunze J, et al. Elevated asymmetric dimethylarginine levels predict short- and long-term mortality risk in critically ill patients. J Crit Care. 2013;28(6):947-953.

20. Winkler MS, Kluge S, Holzmann M, et al. Markers of nitric oxide are associated with sepsis severity: an observational study. Crit Care. 2017;21(1):189.

21. Luft T, Dietrich S, Falk C, et al. Steroidrefractory GVHD: T-cell attack within a vulnerable endothelial system. Blood. 2011;118(6):1685-1692

22. Inamoto $Y$, Ito $M$, Suzuki $R$, et al. Clinicopathological manifestations and treatment of intestinal transplant-associated microangiopathy. Bone Marrow Transplant. 2009;44(1):43-49.

23. Lindås R, Tvedt TH, Hatfield KJ, Reikvam $\mathrm{H}$, Bruserud $\mathrm{O}$. Preconditioning serum levels of endothelial cell-derived molecules and the risk of posttransplant complications in patients treated with allogeneic stem cell transplantation. J Transplant. 2014;2014: 404096.

24. Martin FA, Murphy RP, Cummins PM. Thrombomodulin and the vascular endothelium: insights into functional, regulatory, and therapeutic aspects. Am J Physiol Heart Circ Physiol. 2013;304(12):H1585-1597.

25. Jefferis BJ, Papacosta O, Owen CG, et al. Interleukin 18 and coronary heart disease: prospective study and systematic review. Atherosclerosis. 2011;217(1):227-233.

26. Durpès MC, Morin C, Paquin-Veillet J, et al. PKC- $\beta$ activation inhibits IL-18-binding protein causing endothelial dysfunction and diabetic atherosclerosis. Cardiovasc Res. 2015;106(2):303-313.

27. Kocak H, Oner-Iyidogan Y, Gurdol F, Oner P, Esin D. Serum asymmetric dimethylarginine and nitric oxide levels in obese postmenopausal women. I Clin Lab Anal. 2011;25(3):174-178

28. Cvetković T, Veličković-Radovanović $R$, Stojanović $\mathrm{D}$, et al. Oxidative and nitrosative stress in stable renal transplant recipients with respect to the immunosuppression protocol- differences or similarities? J Med Biochem. 2015;34(3):295-303.

29. Knowles RG, Moncada S. Nitric oxide syn- thases in mammals. Biochem J. 1994;298(Pt 2):249-258

30. Lind M, Hayes A, Caprnda M, et al Inducible nitric oxide synthase: good or bad? Biomed Pharmacother. 2017:93:370375.

31. Suda O, Tsutsui M, Morishita T, et al Asymmetric dimethylarginine produces vascular lesions in endothelial nitric oxide synthase-deficient mice: involvement of reninangiotensin system and oxidative stress Arterioscler Thromb Vasc Biol. 2004;24(9): 1682-1688.

32. Smith CL, Anthony S, Hubank M, Leiper $\mathrm{JM}$, Vallance P. Effects of ADMA upon gene expression: an insight into the pathophysiological significance of raised plasma ADMA. PLoS Med. 2005;2(10):e264.

33. Németh B, Ajtay Z, Hejel L, et al. The issue of plasma asymmetric dimethylarginine reference range - a systematic review and meta-analysis. PLoS One. 2017;12(5): e0177493.

34. Tain YL, Hsu CN. Toxic Dimethylarginines Asymmetric dimethylarginine (ADMA) and symmetric dimethylarginine (SDMA). Toxins (Basel). 2017;9(3):E92.

35. McCarty MF. Asymmetric dimethylarginine is a well established mediating risk factor for cardiovascular morbidity and mortalityshould patients with elevated levels be supplemented with citrulline? Healthcare (Basel). 2016;4(3):E40

36. Allerton TD, Proctor DN, Stephens JM Dugas TR, Spielmann G, Irving BA. 1-citrulline supplementation: impact on cardiometabolic health. Nutrients. 2018;10 (7):E921.

37. Atsuta $\mathrm{Y}$, Hirakawa A, Nakasone $\mathrm{H}$, et al Late mortality and causes of death among long-term survivors after allogeneic stem cell transplantation. Biol Blood Marrow Transplant. 2016;22(9):1702-1709.

38. Tichelli A, Passweg J, Wójcik D, et al. Late cardiovascular events after allogeneic hematopoietic stem cell transplantation: a retrospective multicenter study of the Late Effects Working Party of the European Group for Blood and Marrow Transplantation. Haematologica. 2008;93 (8):1203-1210.

39. Cooke KR, Jannin A, Ho V. The contribution of endothelial activation and injury to endorgan toxicity following allogeneic hematopoietic stem cell transplantation. Biol Blood Marrow Transplant. 2008;14(1 Suppl 1):23-32.

40. Tichelli A, Gratwohl A. Vascular endothelium as 'novel' target of graft-versus-host disease. Best Pract Res Clin Haematol. 2008;21(2):139-148

41. Gratwohl A, Stern M, Brand R, et al Risk score for outcome after allogeneic hematopoietic stem cell transplantation: a retrospective analysis. Cancer. 2009;115(20): 4715-4726

42. Bacigalupo A, Ballen K, Rizzo D, et al. Defining the intensity of conditioning regimens: working definitions. Biol Blood Marrow Transplant. 2009;15(12):1628-1633 